\title{
Familial migraine with aura is associated with a mutation in the TRESK potassium channel
}

$\Lambda$ mutation in the gene encoding the two-pore domain potassium channel TRESK (TWIK-related spinal cord potassium channel) could be an underlying cause of a common hereditary form of migraine with aura, new research published in Nature Medicine indicates. The findings are likely to have implications both for understanding migraine pathogenesis and for guiding the development of new treatment strategies.

In an international collaboration involving teams in Canada, the UK, Portugal and Australia, Ronald Lafrenière, Zameel Cader, Guy Rouleau and colleagues sequenced 150 ion channel genes in a panel of 360 unrelated individuals, including 110 patients with migraine. "We selected migraine with aura and chose probands with a strong family history of migraine to favor cases likely to be caused by deleterious coding mutations in ion channel genes," explains Rouleau.

The investigators decided to focus their attention on the TRESK-encoding gene KCNK18, for two reasons. First, TRESK modulates neuronal excitability and seems to be involved in pain pathways. Second,

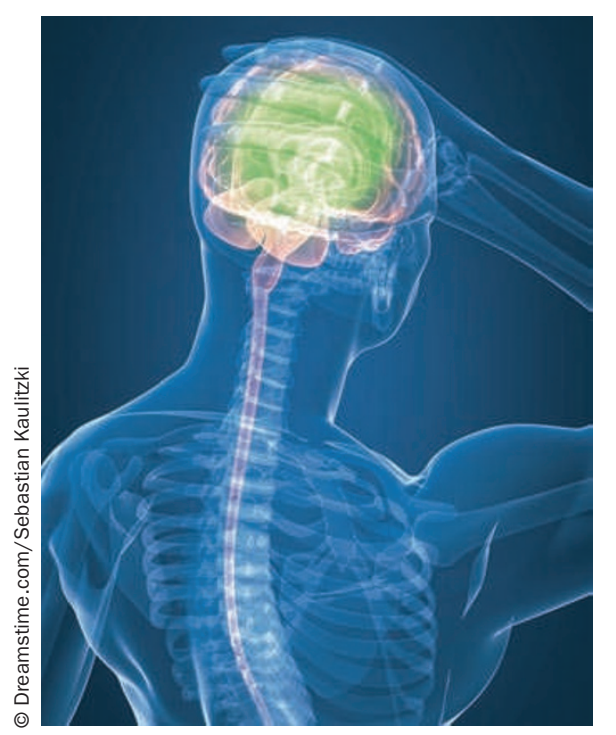

this channel is activated by volatile anesthetics such as halothane, which have been shown to inhibit cortical spreading depression-a key mechanism in the generation of the migraine aura.

"In one migraine proband we identified a 2 bp deletion in the KCNK18 gene, which was predicted to cause a frameshift mutation leading to premature truncation of the TRESK channel," says Lafrenière. The patient belonged to a large multigenerational pedigree, several members of which were affected by a similar form of typical migraine with visual aura. The frameshift mutation, denoted F139WfsX24, was found to cosegregate precisely with the migraine phenotype within this pedigree.

Studies in mouse and human tissue revealed that the KCNK18 transcript and the TRESK protein are expressed in the trigeminal ganglia, which are thought to play a central part in migraine generation. This expression pattern further supports a causative role for the F139WfsX24 mutation in familial migraine with aura.

Next, the researchers performed experiments in Xenopus oocytes to examine the electrophysiological properties of the truncated form of TRESK. They found that the F139WfsX24 mutation caused a complete loss of channel function and, moreover, the mutant channel subunits reduced the activity of the wild-type channel via a dominant-negative mechanism.

The results of this study suggest a possible new approach to the treatment of migraine with aura. "We would expect that the TRESK mutation would make the trigeminal neurons more easily excitable," says Lafrenière. "If we can increase TRESK activity, this might help diminish the excitability of these neurons, and may lessen migraine severity or incidence."

The researchers highlight several advantages of a TRESK-targeting strategy. First, the actions of volatile anesthetics on TRESK might provide clues to mechanisms that could be harnessed therapeutically. Second, given that TRESK is expressed in the trigeminal ganglia, drugs designed to modulate its activity would not necessarily need to cross the blood-brain barrier. Third, TRESK is expressed in a small subset of neurons and its gene has no close paralogs, so development of highly specific drugs with few side effects should be feasible.

\section{4 ...the F139WfsX24 mutation caused a complete loss of channel function... 77}

KCNK18 is not the first gene to be implicated in a hereditary form of migraine-previous studies have linked mutations in CACNA1A, ATP1A2 and $S C N 1 A$ to familial hemiplegic migraine. As Lafrenière points out, however, "our discovery links, for the first time, a common form of migraine to a genetic mutation."

The investigators plan to build on their research by screening other individuals with migraine and their families to determine the prevalence of TRESK mutations. According to Cader, "our findings suggest a whole new pathwaynamely, other members of the two-pore potassium channel family and other ion channels in trigeminal ganglion cells-that could be explored for involvement in migraine and for developing new drugs."

Heather Wood

Original article Lafrenière, R. G. et al. A dominant-negative
mutation in the TRESK potassium channel is linked to
familial migraine with aura. Nat. Med. 16,1157-1160
(2010)
Further reading Chae,Y. J. et al. Discrete change in volatile
anesthetic sensitivity in mice with inactivated tandem
pore potassium ion channel TRESK. Anesthesiology
doi:10.1097/ALN.0b013e3181f90ca5

